\title{
ORIGINAL RESEARCH \\ Cilostazol Prevents Progression of Asymptomatic Carotid Artery Stenosis in Patients with Contralateral Carotid Artery Stenting
}

T. Kato

H. Sakai

T. Takagi

Y. Nishimura

BACKGROUND AND PURPOSE: The progression of atherosclerosis is related to various factors. Although antiplatelet therapy is used for the management of acute ischemic stroke and for the prevention of recurrent stroke, the antiplatelet agent cilostazol may also reduce restenosis after stent implantation in any vessel. This study was performed to assess the impact of cilostazol on plaque progression in the carotid artery contralateral to a stented artery.

MATERIALS AND METHODS: Ninety-five patients who underwent contralateral CAS who also had ipsilateral 0\%-79\% ICS were enrolled. ICS was assessed by duplex sonography every 6 months and by MR imaging/angiography, and digital subtraction angiography if necessary, every 12 months according to the NASCET method. Patient age, sex, past history, and perioperative medical conditions were recorded.

RESULTS: While $22.1 \%$ of patients experienced disease progression, symptomatic ipsilateral stroke occurred in only $1.1 \%$ of patients over $36.2 \pm 18.8$ months. On multivariate analysis, precarotid stenosis (HR per 10\% increase, 2.08; 95\% Cl, 1.43-3.05; $P<.001$ ) and cilostazol use (HR 0.16; 95\% $\mathrm{Cl}, 0.03-0.85 ; P=.03$ ) were independent predictors for the progression of ICS.

CONCLUSIONS: A higher degree of initial stenosis is associated with progression of asymptomatic ICS. Cilostazol may reduce the rate of disease progression in patients with asymptomatic ICS.

ABBREVIATIONS: CAD, coronary artery disease; CAS, carotid artery stenting; CEA, carotid endarterectomy; $\mathrm{Cl}$, confidence interval; CVD, cerebrovascular disease; HR, hazard ratio; ICS, internal carotid artery stenosis; PAD, peripheral artery disease

$\mathbf{L}$ arge randomized controlled clinical trials have shown that CEA is the "gold standard" for the management of ICS, both in symptomatic and in asymptomatic patients. ${ }^{1-4}$ However, CAS placement may be an appropriate alternative therapy for patients with ICS. While the SAPPHIRE trial showed that CAS was noninferior to CEA in patients with high surgical risk factors, ${ }^{5} 3$ other trials failed to prove noninferiority of CAS compared with CEA. ${ }^{6-8}$ Furthermore, only 1 randomized controlled clinical trial found that CEA and CAS are comparable based on major vascular events in terms of safe and effective stroke prevention in patients without high surgical risk factors. ${ }^{9}$ ICS is a major risk factor for stroke; therefore, treatments to reduce the long-term risk of stroke, including medical management, CEA, and CAS, are important for patients with this condition.

Although the incidence of progression of asymptomatic carotid artery disease after contralateral CEA has been described in studies using duplex sonography, the definition of progression varies among these studies. ${ }^{10-13}$ Patients who undergo CAS are given antiplatelet agents in addition to other antiatherosclerotic medications. Antiplatelet agents are effec-

Received August 9, 2011; accepted after revision October 18.

From the Department of Neurosurgery, National Hospital Organization, Toyohashi Medical Center, Toyohashi City, Aichi, Japan.

Please address correspondence to Takayuki Kato, Department of Neurosurgery, National Hospital Organization, Toyohashi Medical Center, 50 Hamamichigami, Imure-cho, Toyohashi City, Aichi, Japan; e-mail: ttaka1010@gmail.com

Indicates open access to non-subscribers at www.ajnr.org

http://dx.doi.org/10.3174/ajnr.A2955 tive for the reduction of stent thrombosis after CAS and for the reduction of the risk of vascular events in other arterial beds, which provides a rationale for their long-term use in these patients. ${ }^{14}$ In patients with coronary and peripheral artery disease, cilostazol has been shown to decrease restenosis and revascularization after catheter intervention. ${ }^{15-26}$ Furthermore, some previous reports suggested that cilostazol reduced restenosis after CEA and CAS. ${ }^{27-29}$ However, no study has investigated whether cilostazol can prevent progression of stenosis in the asymptomatic non-stent-implanted carotid artery after contralateral revascularization. Thus, the goal of this study is to identify predictors of progression of ICS after contralateral CAS.

\section{Materials and Methods}

\section{Patients and Techniques}

Between November 2001 and May 2010, all consecutive patients who underwent successful CAS for ICS in the Department of Neurosurgery at the National Hospital Organization Toyohashi Medical Center were enrolled in this retrospective analysis. Inclusion criteria were patients who could be followed for at least 12 months and who could be assessed for changes of asymptomatic carotid artery stenosis after contralateral CAS. Patient age, sex, past history related to atherosclerosis, and perioperative medical conditions were recorded. Patients were excluded if they had a history of previous CEA, irradiation to the cervical portion of the carotid artery, or traumatic dissection.

All patients were screened with preoperative duplex sonography and MR imaging/angiography, followed by DSA at the time of the procedure, to ascertain whether lesions were appropriate for CAS and to assess the severity of stenosis on the opposite side. The criteria for 
CAS were stenosis greater than $80 \%$ in asymptomatic lesions and greater than $50 \%$ in symptomatic lesions, as per the NASCET method, and CAS was performed using our standard procedure, which has been previously described. ${ }^{30}$ Patients were premedicated with dual antiplatelet therapy in some combination of aspirin (100 $\mathrm{mg} /$ day), ticlopidine (200 mg/day), clopidogrel $(75 \mathrm{mg} /$ day $)$, or cilostazol (200 mg/day), with the choice of agents at the discretion of the attending physicians. Perioperative dual antiplatelet therapy was continued within 1-3 months after CAS, at which point single-agent antiplatelet therapy was continued. In cases of patients with prior $\mathrm{CAD}$ or $\mathrm{PAD}$, dual antiplatelet therapy was continued if necessary.

All patients were assessed every 6 months using duplex sonography by well-trained technicians and every 12 months by MR imaging/ angiography. Patients were instructed to inform their physician if any new symptoms developed after hospital discharge. All new neurologic events were confirmed by an independent neurologist, and brain MR imaging/angiography was performed if any change in neurologic status was found. Furthermore, if new cervical bruit was present on examination, then duplex sonography was performed at each assessment. MR imaging/angiography was also performed when ICS $\geq 50 \%$ was suspected, and DSA was performed when ICS $\geq 80 \%$ was suspected. A $50 \%$ and $80 \%$ ICS was indicated by a peak systolic velocity on duplex sonography of $150 \mathrm{~cm} / \mathrm{s}$ and $230 \mathrm{~cm} / \mathrm{s}$, respectively. ${ }^{31}$ The initial grade of stenosis after contralateral CAS was classified as follows: A, 0\%-49\% stenosis; B, 50\%-79\% stenosis; C, 80\%-99\% stenosis and occlusion. An 80\%-99\% stenosis was an indication for vascular reconstructive surgery, so these patients were excluded from this study. We determined any progression, if the category was advanced to a higher category of stenosis, by comparing serial assessments.

Values are presented as the mean $\pm \mathrm{SD}$. Categoric variables were analyzed by the $\chi^{2}$ or Fisher exact test, as appropriate. Continuous variables with normal distributions were analyzed by the Student $t$ test, and those with non-normal distributions were analyzed by the Mann-Whitney $U$ test. Univariate and multivariate analyses were performed to determine which factors correlated with the progression of asymptomatic ICS. Factors related to the progression of asymptomatic ICS were identified by univariate regression analysis $(P<.20)$ in an exploratory manner, and multivariate logistic regression analysis was performed by age and sex in addition to the extracted factors. Analysis of time to the progression of asymptomatic ICS was based on Kaplan-Meier curves. A probability value $<.05$ was considered statistically significant. All statistical analyses were performed using PASW statistics, version 18 (SPSS Japan, Tokyo, Japan).

\section{Results}

A total of 125 consecutive patients who underwent CAS and who were followed for at least 12 months were included in this study. Nine patients who initially planned to undergo bilateral CAS because of bilateral severe ICS, and 7 patients who had contralateral internal carotid artery occlusion, were excluded. Another 14 patients were excluded because of transfer to another hospital or death within 1 year. Therefore, 95 patients were enrolled in this study. Mean patient age was $73.0 \pm 7.2$ years (range $57-88$ years), and there were 84 (88.4\%) male patients. The mean follow-up period was $36.2 \pm 18.8$ months (range 12-95 months). There were 68 (71.6\%) patients with hypertension, $28(29.5 \%)$ patients with diabetes mellitus, 35 (36.8\%) patients with hypercholesterolemia, 43 (45.3\%) patients with CAD, and $22(23.2 \%)$ patients who were currently smoking. Forty-six (48.4\%) patients experienced symptom-

\begin{tabular}{|c|c|c|c|c|}
\hline Variable & Total & $\begin{array}{c}\text { Stable } \\
(n=74)\end{array}$ & $\begin{array}{c}\text { Progression } \\
(n=21)\end{array}$ & $P$ \\
\hline Age (years) & 73.0 & 72.9 & 73.2 & 0.84 \\
\hline Male (\%) & 88.4 & 87.8 & 90.5 & 1.00 \\
\hline Enrollment period (months) & 36.2 & 34.6 & 41.6 & 0.12 \\
\hline Hypertension (\%) & 71.6 & 71.6 & 71.4 & 1.00 \\
\hline Diabetes mellitus (\%) & 29.5 & 28.4 & 33.3 & 0.79 \\
\hline Hypercholesterolemia (\%) & 36.8 & 36.5 & 38.1 & 1.00 \\
\hline Coronary artery disease (\%) & 45.3 & 43.2 & 52.4 & 0.62 \\
\hline Current smoking (\%) & 23.2 & 23.0 & 23.8 & 1.00 \\
\hline Symptomatic before CAS (\%) & 48.4 & 44.6 & 61.9 & 0.22 \\
\hline
\end{tabular}

\begin{tabular}{lccc}
\hline \multicolumn{4}{l}{ Table 2: Summary of antiplatelet therapy } \\
\hline & Stable & Progression & \% Progression \\
\hline AS (+),$n=62$ & 46 & 16 & 25.8 \\
AS $(-), n=33$ & 28 & 5 & 15.2 \\
TI (+), $n=26$ & 14 & 12 & 46.2 \\
TI (-), $n=69$ & 60 & 9 & 13.0 \\
CL (+), $n=26$ & 23 & 3 & 11.5 \\
CL (-), $n=69$ & 51 & 18 & 26.1 \\
CIL (+), $n=29$ & 27 & 2 & 6.9 \\
CIL (-), $n=66$ & 47 & 19 & 28.8 \\
\hline
\end{tabular}

Note:-The progression of carotid artery stenosis was significantly less in patients with cilostazol use compared with patients without cilostazol use $(P=.03)$. AS indicates aspirin TI, ticlopidine; $\mathrm{CL}$, clopidogrel; CIL, cilostazol. Forty-seven patients received single antiplatelet therapy (AS 16, TI 5, CL 14, CIL 12), 48 patients received dual antiplatelet therapy (AS + TI 20, AS+CL 11, AS + CIL 15, TI+CIL 1, CL+CIL 1). The progression of carotid artery stenosis was significantly less in patients with cilostazol use compared with patients without cilostazol use $(P=.03)$.

atic ischemic neurologic events before contralateral CAS. According to our grading system, 85 patients were categorized as group A (0\%-49\% stenosis) and another 10 patients were categorized as group B (50\%-79\% stenosis). During the follow-up period, 12 (14.1\%) patients progressed from group A to group B, and $4(4.7 \%)$ patients progressed from group A to group C, while $5(50.0 \%)$ patients progressed from group B to group C. In all cases, 21 (22.1\%) patients revealed progression during a period of 36.2 months. One (1.1\%) patient experienced symptomatic ipsilateral stroke, and CAS was performed for ipsilateral ICS in 7 (7.4\%) patients. Age, sex, follow-up period, and risk factors for atherosclerosis were similar comparing patients who experienced progression and those who did not. Baseline characteristics among these subgroups are summarized in Table 1.

A summary of antiplatelet therapy is shown in Table 2 . Aspirin was used in 62 patients, ticlopidine was used in 26 patients, clopidogrel was used in 26 patients, and cilostazol was used in 29 patients. The progression of carotid artery stenosis was significantly fewer in patients with cilostazol use compared with patients without cilostazol use $(6.9 \%$ versus $28.8 \% ; P=.03$ ).

Table 3 shows the univariate and multivariate associations of baseline characteristics, past histories, and perioperative status with the progression of carotid artery stenosis after contralateral CAS. In the initial univariate analyses, the following variables were selected for inclusion in the multivariable models: enrollment period $(P=.14)$, precarotid stenosis $(P<$ $.001)$, symptomatic before CAS $(P=.17)$, cilostazol use $(P=$ $.02)$, and restenosis after CAS $(P=.03)$. In the age- and sexadjusted final multivariate model, the 2 variables identified as 
Table 3: Factors independently associated with the progression of carotid artery stenosis after contralateral carotid artery stenting on multivariate logistic regression models

\begin{tabular}{|c|c|c|c|c|}
\hline \multirow[b]{2}{*}{ Variable } & \multicolumn{2}{|c|}{ Univariate Analyses } & \multicolumn{2}{|c|}{ Multivariate Analyses } \\
\hline & HR $(95 \% \mathrm{Cl})$ & $P$ & HR $(95 \% \mathrm{Cl})$ & $P$ \\
\hline$\overline{\mathrm{Age}^{\mathrm{a}}}$ & $1.12(0.59-2.12)$ & .73 & & \\
\hline Male & $1.32(0.26-6.62)$ & .74 & & \\
\hline Enrollment period & $1.02(0.99-1.04)$ & .14 & & \\
\hline Precarotid stenosis ${ }^{b}$ & $2.02(1.42-2.87)$ & $<.001$ & $2.08(1.43-3.05)$ & $<.001$ \\
\hline Hypertension & $0.99(0.34-2.90)$ & .99 & & \\
\hline Diabetes mellitus & $1.26(0.45-3.57)$ & .66 & & \\
\hline Hypercholesterolemia & $1.07(0.39-2.91)$ & .89 & & \\
\hline Coronary artery disease & $1.44(0.55-3.82)$ & .46 & & \\
\hline Current smoking & $1.05(0.34-3.28)$ & .94 & & \\
\hline Symptomatic before CAS & $2.02(0.75-5.45)$ & .17 & & \\
\hline Cilostazol use & $0.18(0.04-0.85)$ & .02 & $0.16(0.03-0.85)$ & .03 \\
\hline Restenosis after CAS & $4.31(1.11-16.70)$ & .03 & & \\
\hline
\end{tabular}

a Per 10-year increase

b Per $10 \%$ increase.

\begin{tabular}{lccc}
\hline \multicolumn{4}{l}{ Table 4: Rate of progression of carotid artery stenosis } \\
\hline & $\begin{array}{c}\text { Cilostazol } \\
(+)\end{array}$ & $\begin{array}{c}\text { Cilostazol } \\
(-)\end{array}$ & $P$ \\
\hline $\begin{array}{l}\text { Stenosis progression in normal } \\
\text { side }\end{array}$ & $6.9 \%(2 / 29)$ & $28.8 \%(19 / 66)$ & 0.03 \\
Restenosis in CAS side & $0 \%(0 / 29)$ & $15.2 \%(10 / 66)$ & 0.03 \\
\hline
\end{tabular}

Note:-We classified the initial grade of stenosis in normal side as follows: A, 0-49\% stenosis; B, 50-79\% stenosis; C, 80-99\% stenosis and occlusion. We determined any progression, if category was advanced to a higher category of stenosis. Restenosis was defined $50 \%$ stenosis or greater.

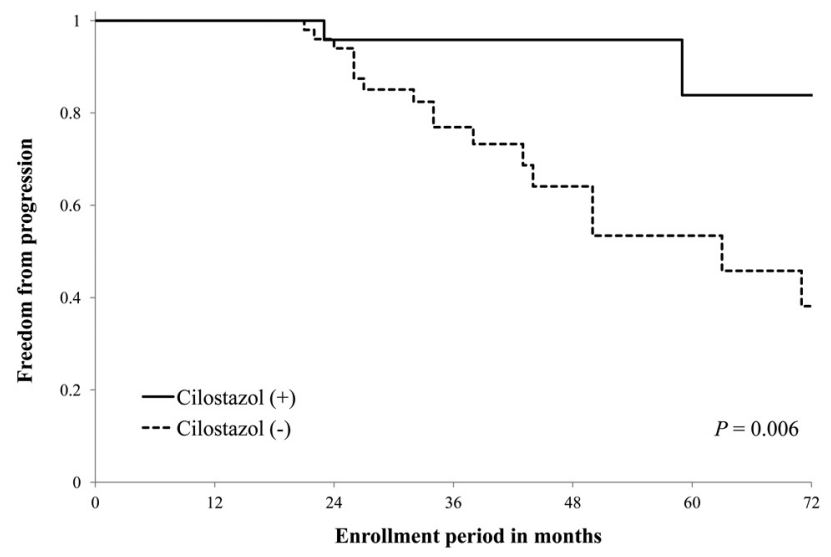

Fig 1. Kaplan-Meier curves showing cumulative freedom from progression of ICS. Freedom from progression of ICS was significantly higher in the cilostazol $(+)$ group compared with cilostazol $(-)$ group $(P=.006)$

independent predictors for the progression of carotid artery stenosis were precarotid stenosis (HR per 10\% increase, 2.08; 95\% CI, $1.43-3.05 ; P<.001$ ) and cilostazol use (HR 0.16; $95 \%$ CI, $0.03-0.85 ; P=.03$ ). In addition, restenosis (defined as $50 \%$ stenosis or greater) after CAS occurred in $10(10.5 \%)$ patients after $32.9 \pm 18.0$ months. The rate of restenosis was $0 \%(0 / 29)$ in patients with cilostazol use and $15.2 \%(10 / 66)$ in patients without cilostazol use $(P=.03$, Table 4$)$.

The Kaplan-Meier curve for freedom from progression of ICS with or without cilostazol use is shown in Fig 1. The cilostazol $(+)$ group had a higher rate of freedom from progression of ICS compared with the cilostazol $(-)$ group $(P=$ $.006)$.

\section{Discussion}

The progression rate of asymptomatic ICS varies from $5.4 \%$ to $16.0 \%$ per year, depending on the definition of stenosis progression used and the population studied. ${ }^{11,12,32-37}$ In the present study, $22.1 \%$ of patients experienced progression of ICS during 36.2 months after contralateral CAS, which is consistent with observations from previous reports. Several studies have described risk factors for ICS progression, including female sex, black race, baseline ICS $50 \%$ or greater, systolic blood pressure $>160 \mathrm{~mm} \mathrm{Hg}$, CAD, smoking, low ankle-brachial pressure index, and echolucent plaques. ${ }^{34-36,38}$ The degree of precarotid stenosis and cilostazol use were independent predictors for progression of carotid artery stenosis after contralateral CAS in multivariate analysis, whereas restenosis after CAS was a significant predictor only within the univariate analysis. Atherosclerosis is a chronic inflammatory disease, ${ }^{39}$ and major factors involved in the progression of atherosclerosis include inflammation of a vulnerable plaque, intraplaque hemorrhage, and injury to vascular endothelial cells. The present study suggests a strong relationship between the degree of initial stenosis and the progression of ICS. Therefore, these patients may benefit from therapies that address atherosclerosis and inflammation, as well as from surgical revascularization in appropriate candidates with severe ICS.

In previous studies, the prevalence of moderate and severe ICS has varied from $0 \%$ to $7.5 \%$ and from $0 \%$ to $3.1 \%$, and the prevalence increased with age and was slightly higher in men. ${ }^{40}$ In Japan, the number of revascularization surgeries for ICS has increased over the past few decades, probably because of the Westernized diet and the development of endovascular therapy. Risk factors for asymptomatic ICS include older age, male sex, cervical bruits, wall thickness on duplex sonography, hypertension, hypercholesterolemia, diabetes mellitus, CAD, $\mathrm{PAD}$, and smoking. ${ }^{37,41-44}$ Within several studies, risk factors in patients with $\mathrm{CAD}, \mathrm{CVD}$, or PAD were remarkably consistent across vascular beds, and this polyvascular disease was the strongest predictor of future ischemic events. ${ }^{45,46}$ In the present study, subjects had already undergone CAS on the other side, and they had a number of core risk factors for atherosclerosis. Despite appropriate control of these risk factors, $22.1 \%$ experienced progression. However, the incidence of symptomatic ipsilateral stroke was only $1.1 \%$ over 36.2 months. In 
patients with asymptomatic ICS of $60 \%$ or greater, ipsilateral ischemic stroke occurred at a rate of $2.3 \%$ per year, and ipsilateral stroke or TIA occurred at a rate of $4.5 \%$ per year. ${ }^{2}$ Similarly, ipsilateral ischemic stroke and all events (stroke, TIA, and amaurosis fugax) occurred $1.5 \%$ and $3.6 \%$ per year, respectively, in patients with ICS of $60 \%$ or greater, whereas ipsilateral ischemic stroke and all events occurred $1.0 \%$ and $2.4 \%$ per year, respectively, in patients with ICS less than $60 \% .^{47}$ In addition to simple luminal stenosis of the artery, plaque components and plaque morphology are important key factors in the occurrence of ischemic events.

In 2001, the average annual rates of ipsilateral stroke among patients receiving medical therapy for vascular disease fell below those of patients who underwent CEA (CEA data were obtained in 1995). However, current medical intervention alone was estimated as at least 3-8 times more cost effective in preventing stroke, and $4-8$ times more cost effective in preventing stroke and TIA compared with medical intervention and surgery as used in past major randomized trials. In addition, there was a $22 \%$ increase in the baseline proportion of patients receiving antiplatelet therapy from 1995 to $2007 .{ }^{48}$ The fall in the rate of ipsilateral ischemic stroke is probably related to pleiotropic medical interventions associated with the use of antihypertensive, hypoglycemic, lipid-lowering, and antiplatelet agents. Several recent studies reported that cilostazol reduced restenosis and repeat revascularization after coronary intervention with either a bare-metal stent or a drug-eluting stent. $^{20,22,26,49,50}$ Similarly, cilostazol reduced restenosis after endovascular therapy in peripheral arteries, ${ }^{15-18}$ and some studies reported the effectiveness of cilostazol in the prevention of restenosis after CAS. In 1 study, restenosis during a 29-month period after CAS occurred in none $(0 /$ 27) of the patients who received cilostazol and in $15.7 \%$ $(11 / 70)$ of patients who did not receive cilostazol. ${ }^{27}$ Independent risk factors for restenosis and revascularization in the treated carotid artery over 30 months after CAS were cilostazol use (OR 0.28; 95\% CI, 0.08-0.95) and stent diameter (OR 0.73; 95\% CI, 0.54-0.99). ${ }^{28}$ Progression of symptomatic intracranial arterial stenosis of the M1 segment in the middle cerebral artery or basilar artery in the cilostazol group was significantly lower than that in the placebo group. ${ }^{51}$ Thus, the present findings that cilostazol use and precarotid stenosis were independent predictors for the progression of asymptomatic ICS, and that the rate of restenosis was lower in patients with cilostazol use, are consistent with observations from previous reports. A recent randomized controlled clinical trial reported that cilostazol was noninferior to aspirin for the prevention of recurrent stroke and that cilostazol was associated with fewer hemorrhagic complications. ${ }^{52}$ Cilostazol has some beneficial effects in addition to its antiplatelet function; these include suppression of neointimal hyperplasia, induction of vasodilation secondary to relaxation of vascular smooth muscle cells, and protection of endothelial cells. ${ }^{53-56}$ Thus, these pluripotent effects of cilostazol probably act to inhibit the progression of ICS.

This study was limited in that it was a nonrandomized, retrospective study. Further, the true effect of cilostazol for asymptomatic ICS remains unknown, as the population in this study underwent contralateral CAS, which may result in some population bias. Therefore, larger prospective multicenter studies are necessary to establish the preventive effect of cilostazol on the progression of true asymptomatic ICS.

\section{Conclusions}

A higher degree of initial stenosis is associated with progression of asymptomatic ICS. Cilostazol may reduce the rate of disease progression in patients with asymptomatic ICS.

\section{References}

1. Beneficial effect of carotid endarterectomy in symptomatic patients with high-grade carotid stenosis. North American Symptomatic Carotid Endarterectomy Trial Collaborators. N Engl J Med 1991;325:445-53

2. Endarterectomy for asymptomatic carotid artery stenosis. Executive Committee for the Asymptomatic Carotid Atherosclerosis Study. JAMA 1995;273:1421-28

3. Randomised trial of endarterectomy for recently symptomatic carotid stenosis: final results of the MRC European Carotid Surgery Trial (ECST). Lancet 1998;351:1379-87

4. Halliday A, Mansfield A, Marro J, et al. Prevention of disabling and fatal strokes by successful carotid endarterectomy in patients without recent neurological symptoms: randomised controlled trial. Lancet 2004;363:1491-502

5. Yadav JS, Wholey MH, Kuntz RE, et al. Protected carotid-artery stenting versus endarterectomy in high-risk patients. N Engl J Med 2004;351:1493-501

6. Mas JL, Chatellier G, Beyssen B, et al. Endarterectomy versus stenting in patients with symptomatic severe carotid stenosis. N Engl J Med 2006;355: $1660-71$

7. Ringleb PA, Allenberg J, Bruckmann H, et al. 30 day results from the SPACE trial of stent-protected angioplasty versus carotid endarterectomy in symptomatic patients: a randomised non-inferiority trial. Lancet 2006;368: $1239-47$

8. Ederle J, Dobson J, Featherstone RL, et al. Carotid artery stenting compared with endarterectomy in patients with symptomatic carotid stenosis (International Carotid Stenting Study): an interim analysis of a randomised controlled trial. Lancet 2010;375:985-97

9. Brott TG, Hobson RW 2nd, Howard G, et al. Stenting versus endarterectomy for treatment of carotid-artery stenosis. N Engl J Med 2010;363:11-23

10. AbuRahma AF, Cook CC, Metz MJ, et al. Natural history of carotid artery stenosis contralateral to endarterectomy: results from two randomized prospective trials. J Vasc Surg 2003;38:1154-61

11. Raman KG, Layne S, Makaroun MS, et al. Disease progression in contralateral carotid artery is common after endarterectomy. J Vasc Surg 2004;39:52-57

12. Ballotta E, Da Giau G, Meneghetti G, et al. Progression of atherosclerosis in asymptomatic carotid arteries after contralateral endarterectomy: a 10-year prospective study. J Vasc Surg 2007;45:516-22

13. Fluri F, Engelter ST, Wasner M, et al. The probability of restenosis, contralateral disease progression, and late neurologic events following carotid endarterectomy: a long-term follow-up study. Cerebrovasc Dis 2008;26:654-58

14. Chaturvedi S, Yadav JS. The role of antiplatelet therapy in carotid stenting for ischemic stroke prevention. Stroke 2006;37:1572-77

15. Iida O, Nanto S, Uematsu M, et al. Cilostazol reduces restenosis after endovascular therapy in patients with femoropopliteal lesions. J Vasc Surg 2008;48:144-49

16. Ishii H, Kumada $\mathrm{Y}$, Toriyama $\mathrm{T}$, et al. Cilostazol improves long-term patency after percutaneous transluminal angioplasty in hemodialysis patients with peripheral artery disease. Clin J Am Soc Nephrol 2008;3:1034-40

17. Soga Y, Yokoi H, Kawasaki T, et al. Efficacy of cilostazol after endovascular therapy for femoropopliteal artery disease in patients with intermittent claudication. J Am Coll Cardiol 2009;53:48-53

18. Soga $\mathrm{Y}$, Iida $\mathrm{O}$, Hirano $\mathrm{K}$, et al. Mid-term clinical outcome and predictors of vessel patency after femoropopliteal stenting with self-expandable nitinol stent. J Vasc Surg 2010;52:608-15

19. Suzuki K, Iida O, Soga Y, et al. Long-term results of the S.M.A.R.T. Con$\operatorname{trol}(\mathrm{TM})$ stent for superficial femoral artery lesions, J-SMART registry. Circ J 2011;75:939-44

20. Douglas JS Jr, Holmes DR Jr, Kereiakes DJ, et al. Coronary stent restenosis in patients treated with cilostazol. Circulation 2005;112:2826-32

21. Ahn Y, Jeong MH, Jeong JW, et al. Randomized comparison of cilostazol vs clopidogrel after drug-eluting stenting in diabetic patients-clilostazol for diabetic patients in drug-eluting stent (CIDES) trial. Circ J 2008;72:35-39

22. Biondi-Zoccai GG, Lotrionte M, Anselmino M, et al. Systematic review and meta-analysis of randomized clinical trials appraising the impact of cilostazol after percutaneous coronary intervention. Am Heart J 2008;155:1081-89

23. Lee SW, Park SW, Kim YH, et al. Drug-eluting stenting followed by cilostazol treatment reduces late restenosis in patients with diabetes mellitus the 
DECLARE-DIABETES Trial (A Randomized Comparison of Triple Antiplatelet Therapy with Dual Antiplatelet Therapy After Drug-Eluting Stent Implantation in Diabetic Patients). J Am Coll Cardiol 2008;51:1181-87

24. Jennings DL, Kalus JS. Addition of cilostazol to aspirin and a thienopyridine for prevention of restenosis after coronary artery stenting: a meta-analysis. J Clin Pharmacol 2010;50:415-21

25. Dihu JB, Abudayyeh I, Saudye HA, et al. Cilostazol: a potential therapeutic option to prevent in-stent restenosis. J Am Coll Cardiol 2011;57:2035-36

26. Lee SW, Park SW, Kim YH, et al. A randomized, double-blind, multicenter comparison study of triple antiplatelet therapy with dual antiplatelet therapy to reduce restenosis after drug-eluting stent implantation in long coronary lesions: results from the DECLARE-LONG II (Drug-Eluting Stenting Followed by Cilostazol Treatment Reduces Late Restenosis in Patients with Long Coronary Lesions) trial. J Am Coll Cardiol 2011;57:1264-70

27. Takigawa T, Matsumaru Y, Hayakawa $M$, et al. Cilostazol reduces restenosis after carotid artery stenting. J Vasc Surg 2010;51:51-56

28. Yamagami H, Sakai N, Matsumaru Y, et al. Periprocedural cilostazol treatment and restenosis after carotid artery stenting: The Retrospective Study of InStent Restenosis after Carotid Artery Stenting (ReSISteR-CAS). J Stroke Cerebrovasc Dis 2012;21:193-99

29. Yoshimoto T, Fujimoto S, Muraki M, et al. Cilostazol may suppress restenosis and new contralateral carotid artery stenosis after carotid endarterectomy. Neurol Med Chir (Tokyo) 2010;50:525-29

30. Kato T, Sakai H, Tsujimoto $\mathrm{M}$, et al. Prolonged carotid sinus reflex is a risk factor for contrast-induced nephropathy following carotid artery stenting. AJNR Am J Neuroradiol 2011;32:441-45

31. Jahromi AS, Cina CS, Liu Y, et al. Sensitivity and specificity of color duplex ultrasound measurement in the estimation of internal carotid artery stenosis: a systematic review and meta-analysis. J Vasc Surg 2005;41:962-72

32. Rockman CB, Riles TS, Lamparello PJ, et al. Natural history and management of the asymptomatic, moderately stenotic internal carotid artery. J Vasc Surg 1997;25:423-31

33. Olin JW, Fonseca C, Childs MB, et al. The natural history of asymptomatic moderate internal carotid artery stenosis by duplex ultrasound. Vasc Med 1998;3:101-08

34. Mansour MA, Littooy FN, Watson WC, et al. Outcome of moderate carotid artery stenosis in patients who are asymptomatic. J Vasc Surg 1999;29:217-25

35. Muluk SC, Muluk VS, Sugimoto H, et al. Progression of asymptomatic carotid stenosis: a natural history study in 1004 patients. J Vasc Surg 1999;29:208-14

36. Liapis C, Kakisis J, Papavassiliou V, et al. Internal carotid artery stenosis: rate of progression. Eur J Vasc Endovasc Surg 2000;19:111-17

37. Cinà CS, Safar HA, Maggisano R, et al. Prevalence and progression of internal carotid artery stenosis in patients with peripheral arterial occlusive disease. J Vasc Surg 2002;36:75-82

38. Jahromi AS, Clase CM, Maggisano R, et al. Progression of internal carotid artery stenosis in patients with peripheral arterial occlusive disease. J Vasc Surg 2009;50:292-98

39. Libby P. Inflammation in atherosclerosis. Nature 2002;420:868-74

40. de Weerd M, Greving JP, Hedblad B, et al. Prevalence of asymptomatic carotid artery stenosis in the general population: an individual participant data metaanalysis. Stroke 2010;41:1294-97

41. Qureshi AI, Janardhan V, Bennett SE, et al. Who should be screened for asymptomatic carotid artery stenosis? Experience from the Western New York Stroke Screening Program. J Neuroimaging 2001;11:105-11

42. Marek J, Mills JL, Harvich J, et al. Utility of routine carotid duplex screening in patients who have claudication. J Vasc Surg 1996;24:572-77; discussion 577-79

43. Fine-Edelstein JS, Wolf PA, O'Leary DH, et al. Precursors of extracranial carotid atherosclerosis in the Framingham Study. Neurology 1994;44:1046-50

44. O'Leary DH, Polak JF, Kronmal RA, et al. Distribution and correlates of sonographically detected carotid artery disease in the Cardiovascular Health Study. The CHS Collaborative Research Group. Stroke 1992;23:1752-60

45. Bhatt DL, Eagle KA, Ohman EM, et al. Comparative determinants of 4-year cardiovascular event rates in stable outpatients at risk of or with atherothrombosis. JAMA 2010;304:1350-57

46. Bhatt DL, Steg PG, Ohman EM, et al. International prevalence, recognition, and treatment of cardiovascular risk factors in outpatients with atherothrombosis. JAMA 2006;295:180-89

47. Nicolaides AN, Kakkos SK, Griffin M, et al. Severity of asymptomatic carotid stenosis and risk of ipsilateral hemispheric ischaemic events: results from the ACSRS study. Eur J Vasc Endovasc Surg 2005;30:275-84

48. Abbott AL. Medical (nonsurgical) intervention alone is now best for prevention of stroke associated with asymptomatic severe carotid stenosis: results of a systematic review and analysis. Stroke 2009;40:e573-83

49. Han Y, Wang S, Li Y, et al. Cilostazol improves long-term outcomes after coronary stent implantation. Am Heart J 2005;150:568

50. Lee SW, Chun KJ, Park SW, et al. Comparison of triple antiplatelet therapy and dual antiplatelet therapy in patients at high risk of restenosis after drug-eluting stent implantation (from the DECLARE-DIABETES and -LONG Trials). Am J Cardiol 2010;105:168-73

51. Kwon SU, Cho YJ, Koo JS, et al. Cilostazol prevents the progression of the symptomatic intracranial arterial stenosis: the multicenter double-blind placebo-controlled trial of cilostazol in symptomatic intracranial arterial stenosis. Stroke 2005;36:782-86

52. Shinohara Y, Katayama Y, Uchiyama S, et al. Cilostazol for prevention of secondary stroke (CSPS 2): an aspirin-controlled, double-blind, randomised non-inferiority trial. Lancet Neurol 2010;9:959-68

53. Ikeda Y, Kikuchi M, Murakami H, et al. Comparison of the inhibitory effects of cilostazol, acetylsalicylic acid and ticlopidine on platelet functions ex vivo. Randomized, double-blind cross-over study. Drug Research 1987;37:563-66

54. Tanaka T, Ishikawa T, Hagiwara M, et al. Effects of cilostazol, a selective cAMP phosphodiesterase inhibitor on the contraction of vascular smooth muscle. Pharmacology 1988;36:313-20

55. Tanaka K, Gotoh F, Fukuuchi Y, et al. Effects of a selective inhibitor of cyclic AMP phosphodiesterase on the pial microcirculation in feline cerebral ischemia. Stroke 1989;20:668-73

56. Ishiguro M, Mishiro K, Fujiwara Y, et al. Phosphodiesterase-III inhibitor prevents hemorrhagic transformation induced by focal cerebral ischemia in mice treated with tPA. PLoS One 2010;5:e15178 